\title{
Origin of high orbital eccentricity and inclination of asteroids
}

\author{
Makiko Nagasawa, Shigeru Ida, and Hidekazu Tanaka \\ Department of Earth and Planetary Sciences, Tokyo Institute of Technology, Meguro-ku, Tokyo 152-8551, Japan
}

(Received October 31, 2000; Revised April 10, 2001; Accepted May 28, 2001)

\begin{abstract}
We investigate the origin of the high orbital eccentricity and inclination of present asteroids. The relative velocity between asteroids corresponding to the observed high eccentricity and inclination is $\sim 5 \mathrm{~km} / \mathrm{sec}$, which is much larger than their surface escape velocity. Thus collisions result in disruption rather than accretion. It is important to clarify when and how their eccentricity and inclination were pumped up, in order to know the environment of asteroidal disruption. The origin of high eccentricity and especially that of inclination have not been well understood yet. We propose sweeping secular resonances due to the gravitational potential change associated with the depletion of the protoplanetary nebula as the excitation mechanism of eccentricity and inclination. We calculated magnitudes of excited eccentricity and inclination through three-dimensional orbital integration. We found that both eccentricity and inclination are excited enough if the protoplanetary nebula is depleted from inside to outside in the region between $5 \mathrm{AU}$ to $10 \mathrm{AU}$ on a timescale $\gtrsim 5 \times 10^{5}$ years. We found that the secular resonances sweep the asteroid belt after the nebula gas is removed from there. Then the asteroidal motion is not affected by gas drag significantly. High velocity collisions between asteroids would occur in a gas-free environment.
\end{abstract}

\section{Introduction}

There are two features of the present asteroid belt that cannot be accounted for by planetary perturbations in the present configuration: (1) both eccentricity $(e)$ and inclination $(i)$ of asteroids are too high; (2) the observationally estimated total mass in the asteroid belt (a few $\times 10^{24} \mathrm{~g}$ ) is only $0.1 \%$ of solid component mass inferred from the minimum mass solar nebula model (Weidenschilling, 1977; Hayashi, 1981).

To consider the collisional evolution of asteroids and production of meteorites, it is important to know when and how their eccentricities and inclinations are excited. In the present paper, we focus on the problem of large orbital excitation, though the second problem should be closely related with the first one.

The random velocities of present asteroids defined by $V_{\text {ran }}=\left(e^{2}+i^{2}\right)^{1 / 2} V_{\text {kep }}$, where $V_{\text {kep }}$ is Keplerian velocity at individual semi-major axes, are shown in the Fig. 1. Inclination $(i)$ is given in radian in this paper. Relative velocities between asteroids are similar to the random velocities. The root mean square of random velocities is about $4.8 \mathrm{~km} / \mathrm{s}$. Above the dotted-broken line, orbits of asteroids with $i=0$ cross the Martian orbit (1.52 AU) and above the dashed line they cross the Jovian orbit (5.20 AU). Asteroids above these lines closely approach the planets and can be strongly scattered. Arrows show the locations of strong resonances. The inclinations of asteroids are excited at the location of the secular resonance called $v_{16}$, and their eccentricities are excited at the location of the secular resonance called $v_{6}$. These secular resonances define the inner edge of

Copy right (C) The Society of Geomagnetism and Earth, Planetary and Space Sciences (SGEPSS); The Seismological Society of Japan; The Volcanological Society of Japan; The Geodetic Society of Japan; The Japanese Society for Planetary Sciences. the main asteroid belt. The locations of secular resonances shown in the Fig. 1 by arrows are those for $i=0, e=0$. The other arrows show the locations of mean-motion resonances. The orbits of asteroids can be largely affected by planetary perturbations only at the narrow regions around resonances, however, $e$ and $i$ (or $V_{\text {ran }}$ ) of asteroids are very high in the entire belt. $V_{\text {ran }}$ and accordingly relative velocity between asteroids are much larger than their surface escape velocities given by $1 \times\left(\mathrm{m} / 10^{25} \mathrm{~g}\right)^{1 / 3} \mathrm{~km} / \mathrm{s}$, where $m$ is mass of asteroid with the density of $3 \mathrm{~g} / \mathrm{cm}^{3}$. Gravity of asteroids accelerates collision velocity up to the surface escape velocity even if $V_{\text {ran }}=0$. If $V_{\text {ran }}>V_{\text {esc }}$, the collision velocities are more than a few times $V_{\text {esc }}$, so that the collision products cannot be bound by their gravity (e.g., Ohtsuki et al., 1993). The collision would rather result in disruption (e.g., Greenberg et al., 1978).

The high eccentricities and inclinations of asteroids cannot result from gravitational scatterings between asteroids. The random velocity acquired by mutual scattering does not exceed $V_{\text {esc }}$, because collision cross section is larger than effective gravitational scattering cross section for $V_{\text {ran }}>V_{\text {esc }}$ (e.g., Safronov, 1969). Even for the largest asteroid, Ceres, its escape velocity is only $\sim 1 \mathrm{~km} / \mathrm{sec}$. It is impossible for mutual gravity of asteroids to excite $V_{\text {ran }}$ up to the present observed value $\sim 5 \mathrm{~km} / \mathrm{sec}$. Wetherill (1989) considered a model that Earth-sized planetesimals were scattered into the asteroid belt and ejected from there after pumping up $e$ and $i$ of asteroids. Earth-sized planetesimals with mass $M$ can pump up $V_{\text {ran }}$ of asteroids up to their surface escape velocity $\simeq 10 \times\left(M / M_{\oplus}\right)^{1 / 3} \mathrm{~km} / \mathrm{s}$. Using statistical methods, Wetherill (1992) showed that after the encounter of asteroidal embryos, remained collisional fragments have a velocity distribution like that of the present asteroids. Petit et al. (2001) considered the effect of planetary embryos on the 


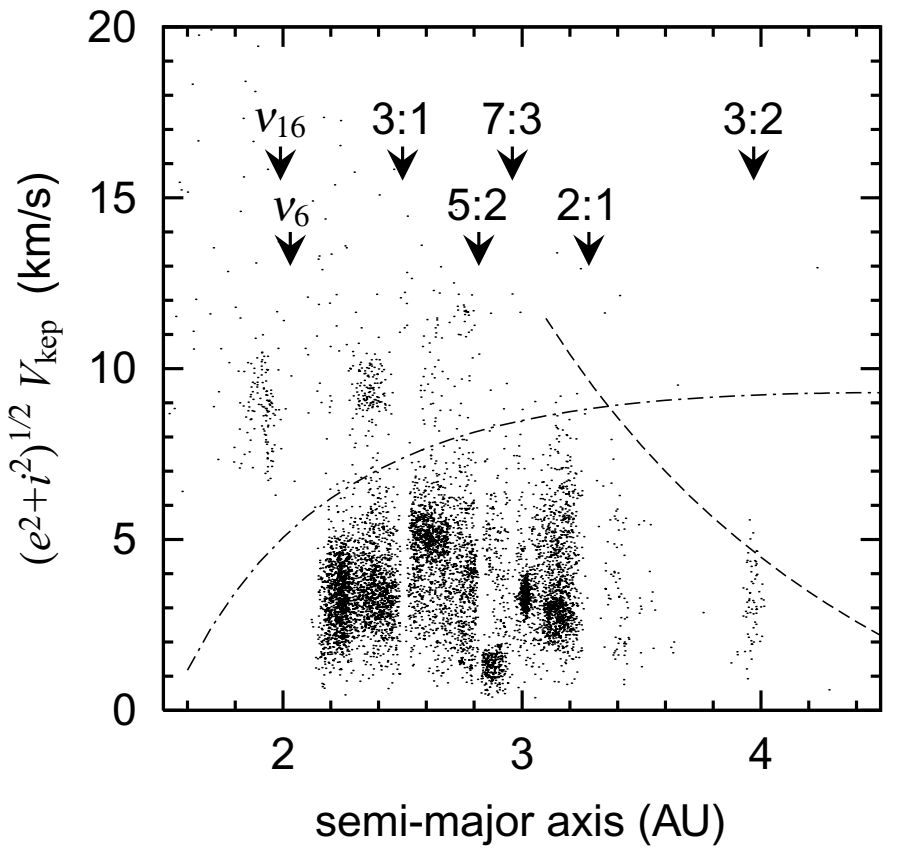

Fig. 1. The random velocities $\left(\left(e^{2}+i^{2}\right)^{1 / 2} V_{\mathrm{kep}}\right)$ versus semi-major axes of the present asteroids. Above the dotted line, orbits of asteroids with $i=0$ cross Jovian orbit, while they cross Martian orbit above the dotted-broken line. The root mean square is about $4.8 \mathrm{~km} / \mathrm{s}$. Arrows show the locations of important mean motion resonances with Jovian orbit and secular resonances. The locations of secular resonances are shown for $i=0, e=0$.

asteroids starting from the stage when the runaway growth of terrestrial planets are ended (Chambers and Wetherill, 1998). They showed that the asteroids stably stay in the asteroid belt with these embryos before Jovian formation. When Jupiter is formed, the orbits of embryos are highly excited by Jovian perturbation and start to affect objects in an entire region of asteroid belt. Most of the asteroids are ejected from asteroid belt (more than $99 \%$ ) by the gravitational stirring by the embryos. The eccentricities and inclinations of remaining asteroids are excited to the observed level. Their model may need optimal timing of formations of terrestrial planets, asteroids, and Jupiter, although we cannot rule out these possibilities of planetary embryos.

Similarly, the high eccentricities and inclinations are not caused by collisional disruptions. Except for a small fraction of fragments, ejected velocities of fragments are significantly smaller than the impact velocities (e.g., Fujiwara and Tsukamoto, 1980). Because collisions are a dissipative process, they damp the eccentricities and inclinations rather than excite them, on average. Actually, members of an asteroid family do not disperse so widely in semi-major axis, eccentricity and inclination.

As suggested in Fig. 1, the secular resonances strongly affect the orbits of asteroids. At present, the resonances are discretely located and pump up $e$ and $i$ in the discrete narrow regions. However, as explained below, secular resonances migrate over the entire asteroid belt when protoplanetary nebula was being depleted. The sweeping of secular resonances is inevitable, because the depletion of the nebula after formation of Jupiter and Saturn is inevitable as explained below.

Secular resonances occur when the proper frequency associated with the longitude of perihelion or that of the as- cending node of an object coincides with one of the eigenfrequencies of planetary (perturber's) precessions. Coincidence of the longitude of perihelion causes excitation of eccentricity, and that of ascending node causes excitation of inclination. When the gravitational potential of the system (nebula potential) changes, secular resonances move, pumping up the eccentricity and the inclination of field objects. This mechanism is called sweeping secular resonances. The importance of the sweeping secular resonances was first suggested by Ward et al. (1976). Jovian and Saturnian gravity causes the secular resonances in the asteroid belt. According to the standard model (e.g., Safronov, 1969; Hayashi et al., 1985), solid cores of Jupiter and Saturn accrete in the protoplanetary disk. When a solid core becomes large enough $\left(\sim 10 M_{\oplus}\right)$, the pressure gradient no longer supports its atmosphere against gravity of the core and gas accretion onto the core starts, which results in formation of gas giants (Mizuno, 1980; Bodenheimer and Pollack, 1986). This means that the nebula gas with at least Jovian mass certainly existed when Jupiter and Saturn accreted at present locations. When the protoplanetary nebula existed, the nebula potential significantly altered locations of the secular resonances because the protoplanetary nebula has more than 10 Jovian masses even in the case of the minimum mass nebula model. This nebula was depleted afterward. There is no nebula gas in the present Solar system. The observations show that the nebula is depleted before a central star becomes a main sequence star (e.g., Strom et al., 1993; Zuckerman et al., 1995). As the nebula was depleted, the secular resonances moved from their initial locations to the present ones.

Heppenheimer (1980) studied the pumping-up of the eccentricities of the asteroids due to the sweeping secular resonances through two-dimensional linear calculations. $\mathrm{He}$ 
found that the eccentricities of asteroids could be pumped up to the present magnitude in the entire region of asteroid belt. Ward (1981) developed techniques to calculate time evolution of secular resonances in a time-dependent axis symmetric potential. Heppenheimer (1980) and Ward (1981) concluded that characteristic timescales of nebula depletion must be shorter than few $\times 10^{4}$ years, in order to avoid excess eccentricities and inclinations of the orbit of terrestrial planets. Including the effect of gas drag, Lecar and Franklin (1997) studied the effect of the sweeping secular resonances in the nebula gas through two-dimensional numerical orbital integration, using a uniform nebula depletion model where nebula surface density decreases with the depletion factor independent of locations in the nebula similar to Heppenheimer (1980) and Ward (1981). They also concluded that the depletion time of $\sim 10^{4}-10^{5}$ years is preferable. If depletion timescale is longer, the asteroids (with size $\lesssim 10 \mathrm{~km}$ ) are removed by gas drag because high eccentricities and inclinations lead to large relative velocities between the particles and nebula gas and accordingly large orbital angular momentum is transferred from the particle to nebula gas through gas drag (Ida and Lin, 1996). On the other hand, if the depletion timescale is shorter, the eccentricities are not pumped up to present values. However, the suggested depletion time $\sim 10^{4}-10^{5}$ years would be inconsistent with lifetime of nebula $\sim 10^{6}-10^{7}$ years inferred from observations of T-Tauri stars (Strom et al., 1993; Zuckerman et al., 1995). Nagasawa et al. (2000) showed that in the case of a non-uniform nebula depletion the required timescale is consistent with $10^{6}-10^{7}$ years. They also found through three-dimensional calculations that the nonuniform depletion model is needed to excite the inclinations of asteroids.

In this paper, we show how random velocities of asteroids are excited during the depletion of the protoplanetary nebula, according to Nagasawa et al. (2000). In Section 2, we explain models we used. In Section 3 we show the results. In Section 4 we give conclusions. The random velocities of asteroids are pumped up to observed magnitude if non-uniform nebula depletion with reasonable timescale $\left(\gtrsim 5 \times 10^{5}\right.$ years) is considered.

\section{Models and Procedures}

We consider a system that consists of Jupiter, Saturn, massless asteroids, and the nebula gas, and simulate the orbital evolution of asteroids. We use the numerical techniques of Nagasawa et al. (2000) for orbital integration. In our simulations, we initially put 38 asteroids (10-100 km sized) on nearly circular, co-planar orbits $(e, i=0.01)$ between 2.05 AU and 3.9 AU with equal interval of 0.05 AU. Initial conditions of Jupiter and Saturn are given by velocities and positions at J2000.0 with the present masses. As for the nebula model, we adopt the minimum mass solar nebula model by Hayashi (1981), in which the distribution of gas density is given by

$$
\begin{aligned}
\rho_{\mathrm{H}} & =1.4 \times 10^{-9}\left(\frac{r}{1 \mathrm{AU}}\right)^{-11 / 4} \\
& \times \exp \left\{-\left(\frac{z}{0.047(r / 1 \mathrm{AU})^{5 / 4}}\right)^{2}\right\}
\end{aligned}
$$

$$
\mathrm{g} / \mathrm{cm}^{3} \quad(a<36 \mathrm{AU}),
$$

where $(r, z)$ are heliocentric cylindrical coordinates. We assume the nebula has a gap with half width $1.233 \mathrm{AU}$ near the orbit of Jupiter (e.g., Lin and Papaloizou, 1993). Nagasawa et al. (2000) showed that non-uniform type of nebula depletion is needed to excite inclinations. We consider the nebula is depleted from inside to outside as

$$
\rho(r, z)= \begin{cases}0 & \text { for } r<r_{\text {edge }}(t) \\ \rho_{\mathrm{H}}(r, z) & \text { for } r>r_{\text {edge }}(t),\end{cases}
$$

with

$$
\dot{r}_{\text {edge }}>0,
$$

where migration speed of the nebula edge, $\dot{r}_{\text {edge }}$, is given by a constant parameter. When $r_{\text {edge }}=5-10 \mathrm{AU}$, secular resonances sweep the asteroid belt. Hence the model we adopt is essentially equivalent to the model that a gap around Jupiter expands with time (Takeuchi et al., 1996). When the nebula is depleted from outside or depleted uniformly, the resonances of inclinations do not sweep in the asteroid belt region (Nagasawa et al., 2000). We also include the effect of hydrodynamic gas drag according to Adachi et al. (1976). As will be shown later, the gas drag does not damp the excited eccentricities and inclinations of asteroids, since secular resonances pass through the asteroid belt and the eccentricities and inclinations are excited after the nebula gas was cleared in the asteroid belt (after $r_{\text {edge }}$ became larger than outer edge of the asteroid belt). In each simulation, orbits are integrated at least for $10^{6}$ years.

\section{Results of Numerical Simulations}

Figure 2 shows an example of the orbital evolution of an asteroid with initial $a=2.35$ AU. The edge of the nebula moves at a constant velocity $\left(\dot{r}_{\text {edge }}=5 \times 10^{-6}\right.$ AU/year). When the secular resonances pass though, the eccentricities and inclinations of asteroids are pumped up. The eccentricities are pumped up three times $\left(t=6 \times 10^{5}\right.$, $1.8 \times 10^{6}$, and $3.4 \times 10^{6}$ years $)$. First two passages are both due to the $v_{5}$ resonance and last passage is due to the $v_{6}$ resonance. The inclinations are pumped up four times $\left(t=5 \times 10^{5}, t=6 \times 10^{5}, 1.8 \times 10^{6}\right.$, and $3.4 \times 10^{6}$ years). These are due to the $v_{16}, v_{15}, v_{15}$, and $v_{16}$ resonance, respectively. First two passages are difficult to distinguish in this figure. At the passage at $t=1.8 \times 10^{6}$ years, $e$ and $i$ are excited more strongly than the other passages. After the nebula depletion, mean values of $i$ and $e$ attain 0.73 and 0.48 , respectively. Figure 3 shows the locations of the secular resonances versus the location of the nebula edge. The lines are calculated according to the Nagasawa et al. (2000). Both eccentricity and inclination resonances sweep occur in the asteroid belt. The resonances pass where the gas was depleted. The $v_{5}, v_{15}$, and $v_{16}$ resonances migrate near Jovian orbit from inside to outside first. These resonances follow behind the nebula edge because of the local change of gravitational potential near the edge. Next, they return and pass through the asteroid belt again from outside to inside, and finally settles down to the present location. The $v_{6}$ resonances occurs after the nebula edge pass though the 


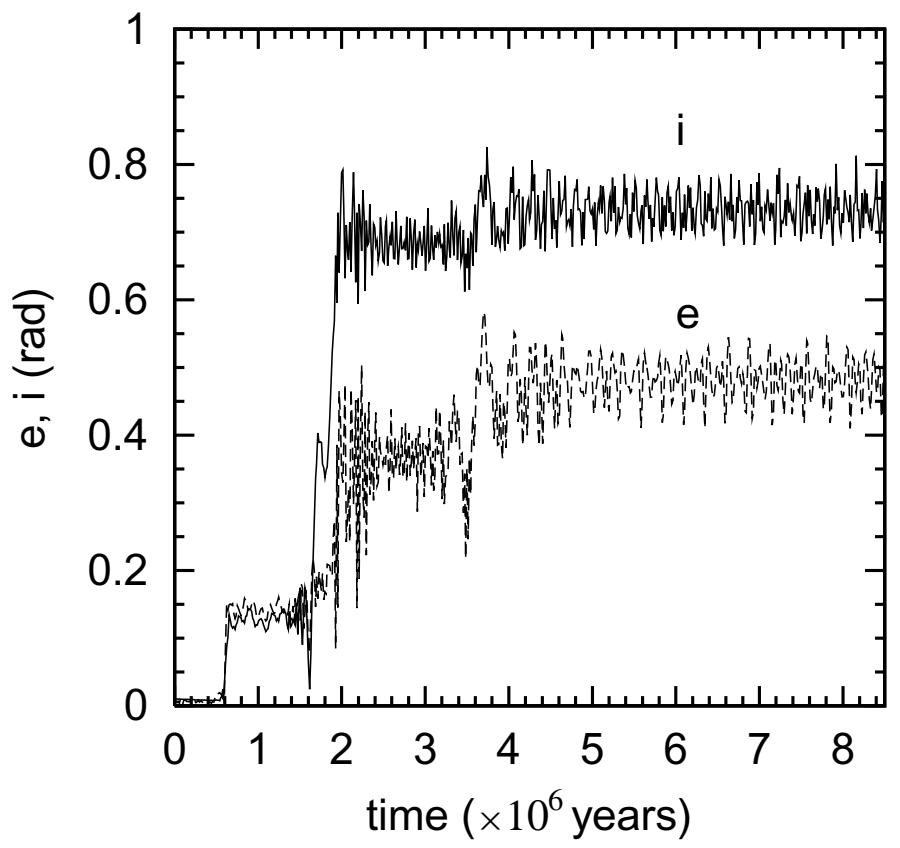

Fig. 2. Orbital evolution of an asteroid with initial $a=2.35 \mathrm{AU}, e, i=0$. The edge velocity $\dot{r}_{\text {edge }}=5 \times 10^{-6} \mathrm{AU} /$ year.

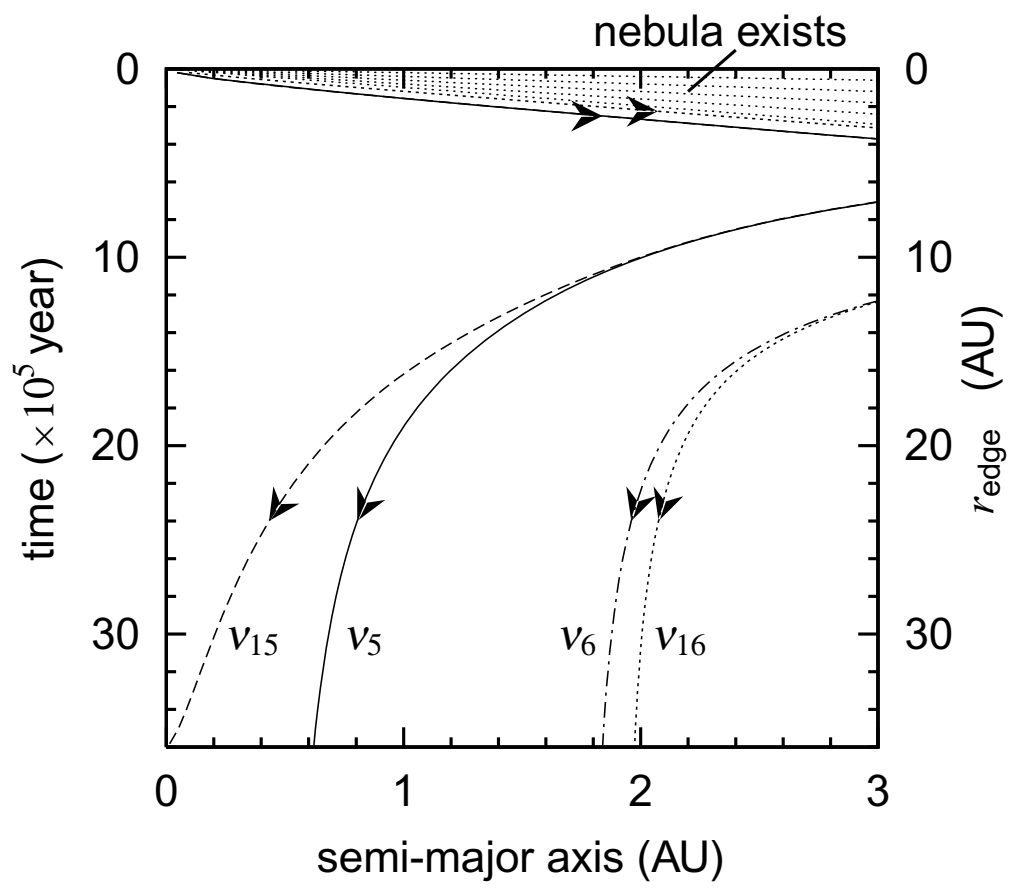

Fig. 3. Locations of the secular resonances $v_{5}, v_{6}, v_{15}$, and $v_{16}$ as functions of the time. The migration speed of nebula edge is $\dot{r}_{\text {edge }}=1 \times 10^{-5} \mathrm{AU} /$ year. The relations between the position of nebula edge $\left(r_{\text {edge }}\right)$ and the locations of the secular resonances do not depend on the migration speed of nebula edge. The shadowed aria shows the region where the nebula is remained.

Saturnian orbit. The $v_{16}$ resonance pass $a=2.35 \mathrm{AU}$ when the nebula edge is at $r_{\text {edge }}=2.5 \mathrm{AU}$ and $r_{\text {edge }}=16.7$ AU. For the edge velocity $\dot{r}_{\text {edge }}=5 \times 10^{-6}$ AU/year, these passage is at $6 \times 10^{5}$ and $3.3 \times 10^{6}$ years (see Fig. 2 ). The results of our simulations show good agreement with the analytical estimates.

Figure 4 shows snapshots of inclination versus semi- major axis of test particles. The edge of the nebula moves at a constant velocity ( $\dot{r}_{\text {edge }}=5 \times 10^{-6}$ AU/year). Horizontal bars show the distance from perihelia $(a(1-e))$ to aphelia $(a(1+e))$. With the horizontal bars, we can also see the excitation of eccentricities. The orbits of particles cross each other when these bars overlap in semi-major axis. The panel of $t=0$ shows the initial state $(e=i=0.01)$. At 


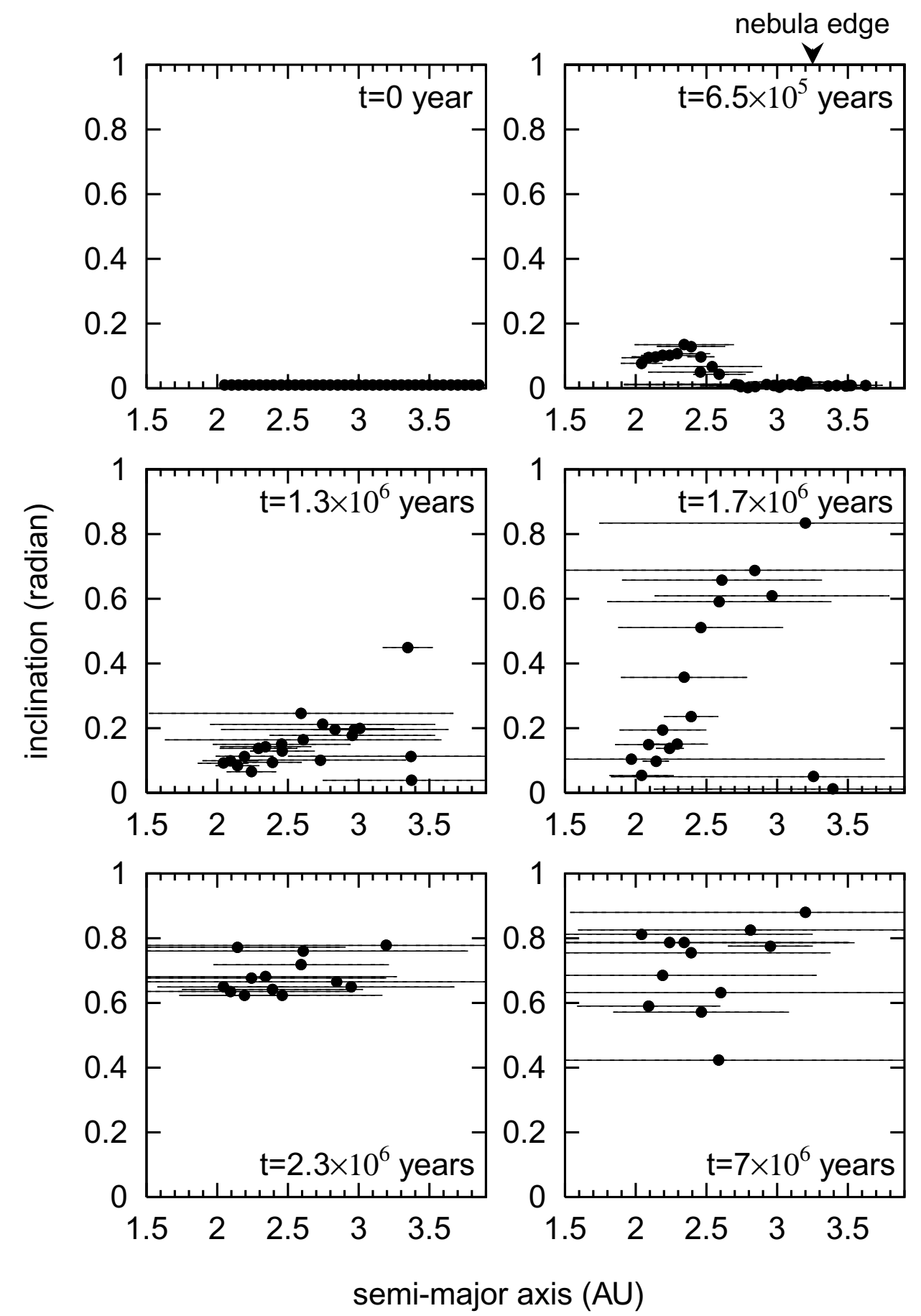

Fig. 4. The evolution of inclinations vs. semi-major axis in the cases that the velocity of nebula edge is $5 \times 10^{-6}$ AU/year. Horizontal bars show radial excursion due to eccentricities, more precisely, the distance from perihelia to aphelia.

$t=6.5 \times 10^{5}$ years, the first secular resonances begin to pass the asteroid belt from inside to outside. Inside 2.6 AU, the inclinations and eccentricities of particles are excited by the resonance passage. In this area, random velocities $\left(V_{\text {ran }}=\right.$ $\left.\left(e^{2}+i^{2}\right)^{1 / 2} V_{\mathrm{kep}}\right)$ are $3-5 \mathrm{~km} / \mathrm{s}$, which already exceeds surface escape velocities $(\sim 1 \mathrm{~km} / \mathrm{s})$ of $1000 \mathrm{~km}$-sized particles. The collision cross sections between particles are larger than scattering cross sections with this speed. The nebula edge passes through a location of $a$ at $2 \times 10^{5}(a / 1 \mathrm{AU})$ years. We found that the resonances pass after the nebula edge passes through (see Fig. 3), i.e., the orbits of asteroids are exited after the nebula is depleted around those asteroids (right upper panel of Fig. 4). Therefore, the gas drag is not effective, and the semi-major axes of particles are not damped by gas drag except at narrow regions of low-order mean motion resonances. Both secular resonances of inclination and eccentricity pass through the asteroid belt three times as seen from Fig. 2. At $t=1.3 \times 10^{6}$ years, the first resonances have passed the asteroid belt. Some particles acquire large enough eccentricity for orbit crossing with Jupiter and are ejected from the asteroid belt by Jovian perturbations. The random velocities of most particles are excited to $\sim 5 \mathrm{~km} / \mathrm{s}$. 


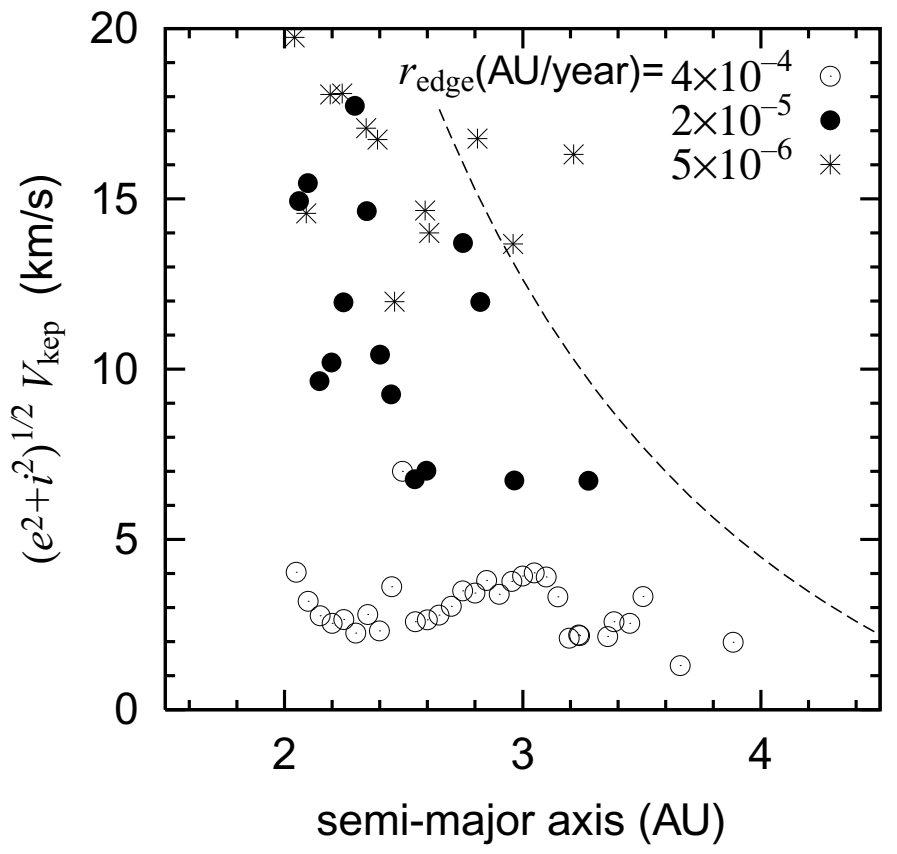

Fig. 5. Final random velocities versus final semi-major axes. Orbital calculations are done for three different velocities of edge $\left(4 \times 10^{-4}, 2 \times 10^{-5}\right.$, and $5 \times 10^{-6} \mathrm{AU} /$ year). Above the dotted line, orbits of asteroids closely approach Jovian orbit and are scattered.

At $t=1.7 \times 10^{6}$ years, the second resonances are passing through the asteroid belt from outside to inside. The second resonances pass over at $t=2.3 \times 10^{6}$ years. The last resonances pass though the asteroid belt from outside to inside at about $t=4 \times 10^{6}$ years. The particles obtain large eccentricities and inclinations. The random velocities are excited up to $20 \mathrm{~km} / \mathrm{s}$. Since the resonances can be either constructive or destructive when eccentricities and inclinations are not zero, final random velocities are spread out to some degree. Although the random velocities of all particles stay at high levels in this simulation, they would be diffused by later mutual collisions.

We performed the simulation in the cases of $\dot{r}_{\text {edge }}=$ $4 \times 10^{-4}, 2 \times 10^{-5}$, and $5 \times 10^{-6}$ AU/year. The compiled results of the final eccentricities and inclinations are shown in Fig. 5. The random velocities are excited to larger values when the nebula edge migrates more slowly. Nagasawa et al. (2000) analytically showed that the excitation level is roughly proportional to $\left(\dot{r}_{\text {edge }}\right)^{-1 / 2}$. The observed random velocities are explained in the cases that $\dot{r}_{\text {edge }}$ is slower than $10^{-5} \mathrm{AU} / \mathrm{year}$. The excitation magnitudes of inclinations depend on the discrepancies between the nebula mid-plane and the planets. In this paper, we fix the nebula mid-plane on the current ecliptic plane. If the nebula mid-plane exactly coincides with the invariant plane of Jupiter and Saturn, the excitation effect is the minimum. In that case, the required $\dot{r}_{\text {edge }}$ to excite the inclinations of asteroids up to the observational level is slower by a factor 10 than in the case that the nebula mid-plane coincides with the elliptic plane.

\section{Conclusions}

We have investigated the evolution of eccentricities and inclinations of asteroids caused by the sweeping secular resonances associated with depletion of the protoplanetary neb- ula. The observed high random velocities (orbital eccentricities and inclinations) of asteroids cannot be explained by planetary perturbations in the present configuration. Sweeping secular resonances can be responsible for the high random velocity. We performed orbital calculations of a system that consists of Sun, Jupiter, Saturn, test particles (corresponding to asteroids), and nebula gas, according to Nagasawa et al. (2000). In order to pump up orbital inclination, the nebula must be depleted non-uniformly, e.g., from inside to outside. We found that random velocities are pumped up high enough to be comparable to the observed magnitude in the entire asteroid belt if the nebula edge migrates from $5 \mathrm{AU}$ to $10 \mathrm{AU}$ on a timescale longer than $5 \times 10^{5}$ years in the case of the inside-out depletion of nebula. In the case of $\dot{r}_{\text {edge }}=5 \times 10^{-6}$ AU/year, random velocities are excited up to $20 \mathrm{~km} / \mathrm{s}$ mostly during when the nebula edge is moving in 5-10 AU.

We found that in this inside-out depletion model, the secular resonances pass through the asteroid belt after nebula gas has already been depleted there. As a result, the particles remain in the asteroid belt without damping of eccentricities and inclinations due to gas drag even if the nebula depletion timescale is as long as the observationally inferred one. Therefore, high velocity collisions between asteroids would have occurred in gas-free environment. The deficiency of total mass in the asteroid belt is still unsolved problem in our mechanism of sweeping secular resonances. Although a timing of erosion is not matter in our model, the mass depletion problem must be solved consistently with observed properties of taxonomic asteroidal families.

Acknowledgments. We acknowledge K. Nakazawa and H. Emori for fruitful discussions. We thank J. Lissauer and S. J. Weidenschilling for careful reading. We used the parallel computer (Silicon Graphics Origin 2000) of Earthquake Information Center of 
Earthquake Research Institute, the University of Tokyo.

\section{References}

Adachi, I., C. Hayashi, and K. Nakazawa, The gas drag effect on the elliptical motion of a solid body in the primordial solar nebula, Prog. Theor. Phys., 56, 1756-1771, 1976

Bodenheimer, P. and J. B. Pollack, Calculations of the accretion and evolution of giant planets: The effects of solid cores, Icarus, 67, 391-408, 1986.

Chambers, J. E. and G. W. Wetherill, Making the Terrestrial planets: Nbody integrations of planetary embryos in three dimensions, Icarus, 136, 304-327, 1998.

Fujiwara, A. and A. Tsukamoto, Experimental study on the velocity of fragments in collisional breakup, Icarus, 44, 142-153, 1980.

Greenberg, R., W. K. Hartmann, C. R. Chapman, and J. F. Wacker, Planetesimals to planets-Numerical simulation of collisional evolution, Icarus, 35, 1-26, 1978

Hayashi, C., Structure of the solar nebula, growth and decay of magnetic fields and effects of magnetic and turbulent viscosities on the nebula, Prog. Theor. Phys. Suppl., 70, 35-53, 1981.

Hayashi, C., K. Nakazawa, and Y. Nakagawa, Formation of the solar system, in Protostars and Planets II, edited by D. C. Black and M. S. Mathews, pp. 1100-1153, Univ. of Arizona Press, Tucson, 1985.

Heppenheimer, T. A., Secular resonances and the origin of eccentricities of Mars and the asteroids, Icarus, 41, 76-88, 1980.

Ida, S. and D. N. C. Lin, Long Term Gas Drag Effect on the Structure of the Asteroid Belt and its Implications for the Solar Nebula, AJ, 112, 1239 1246, 1996.

Lecar, M. and F. Franklin, The Solar Nebula, Secular Resonances, Gas Drag, and the Asteroid Belt, Icarus, 129, 134-146, 1997.

Lin, D. N. C. and J. C. B. Papaloizou, On the tidal interaction between protostellar disks and companions, in Protostars and Planets III, edited by E. H. Levy and J. I. Lunine, pp. 749-835, Univ. of Arizona Press, Tucson, 1993.

Mizuno, H., Formation of the Giant Planets, Prog. Theor. Phys. Suppl., 64, 544-557, 1980.
Nagasawa, M., H. Tanaka, and S. Ida, Orbital Evolution of Asteroids during Depletion of the Solar Nebula, AJ, 119, 1480-1497, 2000.

Ohtsuki, K., S. Ida, Y. Nakagawa, and K. Nakazawa, Planetary accretion in the solar gravitational field, in Protostars and Planets III, edited by E. H. Levy and J. I. Lunine, pp. 1089-1107, Univ. of Arizona Press, Tucson, 1993.

Petit, J. M., A. Morbidelli, and J. Chambers, The primordial excitation and clearing of the asteroid belt, 2001 (preprint).

Safronov, V. S., Evolution of Protoplanetary Cloud and Formation of the Earth and Planets, Nauka, Moscow, NASA Tech. Trans. F-677 1969.

Strom, S. E., S. Edwards, and M. F. Skrutskie, Evolutionary timescales for circumstellar disks associated with intermediate- and solar-type stars, in Protostars and Planets III, edited by E. H. Levy and J. I. Lunine, pp. 837-866, Univ. of Arizona Press, Tucson, 1993.

Takeuchi, T., S. M. Miyama, and D. N. C. Lin, Gap Formation in Protoplanetary Disks, ApJ, 460, 832-847, 1996.

Ward, W. R., Solar nebula dispersal and the stability of the planetary system. I-Scanning secular resonance theory, Icarus, 47, 234-264, 1981

Ward, W. R., G. Colombo, and F. A. Franklin, Secular resonance, solar spin down, and the orbit of Mercury, Icarus, 28, 441-452, 1976.

Weidenschilling, S. J., The distribution of mass in the planetary system and solar nebula, Astrophys. Space Sci., 51, 153-158, 1977.

Wetherill, G. W., Origin of the asteroid belt, in Asteroids II, edited by R. P. Binzel, T. Gehrels, and M. S. Matthews, pp. 661-680, Univ. of Arizona Press, Tucson, 1989.

Wetherill, G. W., An alternative model for the Formation of asteroids, Icarus, 100, 307-325, 1992.

Zuckerman, B., T. Forveille, and J. H. Kastner, Inhibition of Giant Planet Formation by Rapid Gas Depletion around Young Stars, Nature, 373 494-496, 1995.

M. Nagasawa (e-mail: mnagasawa@mail.arc.nasa.gov), S. Ida, and H. Tanaka 